\title{
Suture Materials, 1980s: Properties, Uses, and Abuses
}

\author{
Neil A. Swanson, M.D. and Theodore A. Tromovitch, M.D.
}

Throughout antiquity many materials have been used to approximate wounds. As early as 5000-3000 B.C.E., eyed needles were used to pass suture material through surgical wounds. By 2000 B.C.E. boned needles began usage in a fashion unsurpassed until Renaissance times. Materials connected to these needles included flax, hemp, fascia, hair, linen strips, pig's bristles, reeds, grasses, and other exotic materials available. Tying of locks of scalp hair into knots to close scalp wounds was common during battles and is still employed as a temporary measure in some busy emergency rooms.

Perhaps the most legendary wound closure was the use of the mouth parts of the pincher ant. In all, natural products alone sufficed as suture material until the 1940s. These included silk, cotton, linen-cotton, and catgut. During this decade, nylon and dacron became the first synthetic materials made into suture. Later, polyethylene, polypropylene, polyglycolic acid, and polyglycan 910 were developed and added to the surgeon's armamentarium. For years, the surgeon had no choice but to use silk and catgut to close wounds.

With the advent of modern suture material, one should become familiar with the different characteristics of each so that he may best use the most appropriate product. The qualities of the ideal suture material have been bantered for years. Characteristics of such a suture material include superior tensile strength, good knot security, excellent handling characteristics, minimal, nonallergenic tissue reaction, resistance to infection, and eventual absorption (of absorbable suture products) when tissue repair has reached satisfactory levels. Unfortunately, there is no ideal suture material.

The U.S. Pharmacopeia divides sutures into two groups, the absorbable and the nonabsorbable. In general, absorbable sutures lose tensile strength in less than 60 days; nonabsorbable sutures maintain tensile strength

Address for reprints: Neil A. Swanson, M.D., C-2074 Outpatient Building University of Michigan Medical School, Ann Arbor, MI 48109 .

\author{
From the Department of Dermatology, \\ University of Michigan Medical School, \\ Ann Arbor, Michigan \\ and the Department of Dermatology, \\ University of California Medical School at San Francisco, \\ San Francisco, California
}

for greater than 60 days. Tables 1 and 2 list several different sutures available in each category which are of importance to the dermatologic surgeon. The table further summarizes the important physical characteristics each suture possesses.

\section{Properties of Suture Material ${ }^{1,3}$}

Knot security refers to the ability of the suture to maintain knot strength without slippage. It is important to tie careful square knots to impart a fair test of knot security. Knot security is inversely proportional to the memory of the suture material, and directly proportional to the workability of the suture material. Sutures of high memory are less pliable, maintain their original shape, and can be more difficult with which to work. Knot security can be improved by an extra tie when securing a surgical knot. Examples of sutures with good memory are monofilament nylon and polypropylene.

Tensile strength ${ }^{4}$ is used for both suture material and tissue. It refers to the strength per unit area of tissue or suture. Breaking strength, on the other hand, involves a measurement of strength for unit width and differs from tensile strength. Skin and fascia are tissues of greatest tensile strength, a quality which experimentally increases with age. Theoretically, therefore, suture material need not have greater tensile strength than the would being closed.

Tissue reactivity ${ }^{5}$ has become increasingly important in considering suture material. Biopsy material from wounds after suture implantation has revealed a marked and peaked tissue reaction and two to seven days after 
TABLE 1. The Absorbable Sutures

\begin{tabular}{lrrrr}
\hline \multicolumn{1}{c}{ Type } & $\begin{array}{c}\text { Knot } \\
\text { Security }\end{array}$ & $\begin{array}{c}\text { Tensile } \\
\text { Strength }\end{array}$ & $\begin{array}{c}\text { Wound } \\
\text { Security }(\%)\end{array}$ \\
\hline Gut & + & ++ & $5-7$ da $(50 \%)$ & $10-14$ da $(50 \%)$ \\
Chromic & ++ & ++ & 25 da $(50 \%)$ & +++ \\
Dexon* & ++++ & ++++ & 30 da $(50 \%)$ \\
Vicrylt & +++ & +++ & + \\
\hline
\end{tabular}

* Polyglycolic acid.

+ Polyglycan 910.

implantation. This occurs to some degree with all sutures studied. The information is characterized in the first four days by polymorphonuclear leukocytes, lymphocytes, and monocytes. By the fourth day, mononuclear cells, macrophages, and fibroblasts predominate. After the seventh day, fibrous tissue with chronic inflammation persists. The inflammation is universally present for seven days for all suture products. It then decreases for most nonasorbable sutures, with the monofilament sutures, especially polypropylene, exhibiting the least reactivity. The absorbable sutures, however, differ in their reactivity. Plain catgut maintains an intense reaction until the suture is absorbed, with chromic gut being slightly less reactive. Synthetic polyglycolic acid (PGA) and polyglycan are much less reactive.

Wound security refers to the strength the suture imparts to the wound over time. It is a reflection not only of wound healing, but of the tensile strength, know security, and other characteristics of the suture material. For the absorbable suture, it is a reflection of their less of tensile strength over time. Wound security, therefore, is dependent on both original tensile strength and rate of absorption. Therefore, although some studies show that PGA is absorbed more rapidly than chromic gut, the original tensile strength of PGA is much greater than chromic catgut, thus imparting a much greater wound security to PGA.

\section{Absorbable Sutures}

Catgut $^{6}$ (plain gut) is made from sheep intima and has historically been the longest available and best-known suture; however, its use today is declining. Catgut maintains significant tensile strength for approximately four to five days. Wound security is essentially gone in 14 days. It also exerts the greatest amount of tissue reaction of all the absorbable sutures. Therefore, to place catgut sutures other than for hemostasis in a wound where the skin sutures are going to be removed in a week to 14 days does not make mechanical sense. It is, in a sense, introducing a foreign body which will not add to the strength of the wound once the skin suture has been removed. It can be used on a surface such as the mucosa which heals rapidly and advantage taken of its rapid absorption.

Chromic gut ${ }^{3,6}$ is historically almost as old as catgut. It is catgut which has been treated by exposure to various chromic salts in an attempt to retard absorption in tissue. It has better wound security in that its absorption is somewhat delayed compared with catgut. Some studies have shown that its rate of degradation in tissue is slower than some of the modern absorbable sutures; its tensile strength is much less. It only maintains wound security for about 14 days. After this, wound security markedly diminishes and it is more useful than catgut in wound approximation. Where true wound security is an issue, it

TABLE 2. The Nonabsorbable Sutures

\begin{tabular}{|c|c|c|c|c|c|}
\hline Silk & ++++ & + & + & $+++t$ & ++++ \\
\hline Nurolont & +++ & ++ & ++ & +++ & \\
\hline Nylon & ++ & +++ & +++ & ++ & ++ \\
\hline Prolene $\neq$ & + & ++++ & ++++ & + & + \\
\hline
\end{tabular}

* Multifilament dacron.

+ Braided monofilament nylon.

‡ Polypropylene.

$\S$ Coated braided polyester. 
still is inferior to more modern suture materials. It also imparts a good deal of tissue reaction.

There is one chromic gut suture, manufactured by Davis and Geck, called "mild ophthalmic chromic gut" which has somewhat less reaction in tissue but is absorbs too rapidly for routine use. It is available in a small $(6 / 0)$ size, which can be used as an external skin suture in a special way for the suturing of grafts and blephroplasties in delicate areas where suture removal would present a problem. If the suture line is covered with a piece of tape, the mild ophthalmic usually absorbs within a week, so that removing the tape at that time generally removes all the suture at the same time. This has been observed only for Davis and Geck's mild ophthalmic chromic and not for the other types of mild chromic. Except for the special uses discussed for both catgut and chromic gut suture, they probably should be replaced by more modern materials.

Vicryl 5, 7,8 (polyglycan 910) is a synthetic polymer. Prepared by the copolarization of a mixture of lactide and glycolide. A catalyst, a colorant (if the suture is dyed) and a chemical regulator, are also present in the reaction. The polymer which is formed is then melted and extruded into fine fibers. These fibers are then braided into sutures making this a synthetic braided suture. The braiding provides better handling properties. The synthetic fiber is available in both dyed and clear sutures. The dye is used primarily to enhance suture visibility. The suture possesses an extremely high tensile strength and maintains $50 \%$ of that tensile strength for approximately thirty days. It requires 90 to 120 days for complete dissolution. Its removal is accomplished by hydrolysis without significant phagocytosis. This supposedly causes less tissue inflammatory response than gut sutures. Vicryl has less knot security, so that the placing of knots must be more accurate. Vicryl biologically makes more sense in a wound which will require tensile strength subcutaneously during healing, since the sutures maintain strength for the four to six-week period of collagen formation in the healing process.

Dexon 5, 8-10 (polyglycolic acid) represents another of the modern absorbable sutures. Polyglycolic acid is a high molecular, linear copolymer of glycolic acid. It is extruded into thin filaments and braided into sutures in much the same way as other synthetic fibers. It possesses many of the same qualities as Vicryl. Its knotting characteristics are very similar. It is said that knot security with Dexon is 1.4 times greater than chromic catgut and two times greater than catgut. ${ }^{6}$ It has an extremely high tensile strength and maintains $50 \%$ of tensile strength in tissue for 25 days. Again, all of the suture material generally is totally removed in 90 to 120 days. It also provides a very low tissue reactivity compared to catgut sutures. It is dissolved by autolytic action in the tissues and does not involve phagocytosis. A problem with
Dexon has been that the braided suture has a tendency to "hang up" when attempting to tie knots. The loop on an instrument tie will sometimes lock on the braided structure so that the knot cannot be set exactly where desired. In 1978, a new Dexon was introduced composed of smaller multifilament strands in a tighter weave and this has compensated somewhat for this problem. Dexon and Vicryl sutures have now basically replaced the routine use of catgut sutures in dermatologic surgery.

Coated Vicryl is a new suture which is essentially Vicryl with a Teflon ${ }^{\circledR}$ coating. It has the advantage of much easier slippage through tissue without the braided strands getting caught in wound margins. Its knots also tie more easily and the suture has less memory, thus making it easier to handle. The knots, however, do have more of a tendency to slip and must be placed accurately.

\section{Nonabsorbable $e^{12-14}$}

Silk has been a mainstay suture material for years. It possesses an extremely adequate workability and knot security. It has very little memory, enabling these qualities to be apparent. It does, however, induce most tissue reactions of any nonabsorbable sutures. It imparts the least tensile strength of the nonabsorable sutures, losing $50 \%$ of the strength in one year, and all of its tensile strength by the end of two years. It still is a suture used in key locations with great benefit. Its lack of memory and, therefore, good workability make it useful around the eye, mouth, and orifices. It also can be used on the soles of feet where pressure is being placed.

Mersilene (dacron-polyester) is a braided synthetic suture. Its braided construction offers tying ease, workability, and knot security similar to those of silk. It is present in both dyed and undyed materials which makes its use beneficial for certain areas. It causes less tissue reaction than silk. It can be used in the same areas where silk can be used because of its lack of memory. It has extremely high tensile strength and remains in tissue for more than two years.

Neurolon (braided nylon) consists of fine nylon braided again in an attempt to give tying characteristics closer to those of silk, while retaining the low reactivity of nylon. Surgeons who prefer nylon sutures will find this suture beneficial in areas where lack of memory and easy tying characteristics are of benefit. Its tensile strength is very similar to nylon, and it also can remain in tissue for more than two years. Its tensile strength, however, is less than the tensile strength of polypropylene.

Nylon is a monofilament extruded nylon synthetic material. It has a good deal of memory, thus, know security can be a problem if care is not taken in a proper surgical tie. It has a great deal of tensile strength and provides very adequate wound security for skin. It has much less tissue reaction making it a valuable suture for 
routine use. Monofilament sutures have several advantages over braided sutures. The synthetic monofilament sutures offer the advantage of decreased wound infection compared to braided suture. They also can be used more safely than braided sutures in contaminated wounds. This, coupled with their high tensile strength and low tissue reactivity, make them an excellent cutaneous suture. They can offer more aggravation during the surgical procedure because of their increased memory. The first throw that is placed has a tendency to slip, and this must be accounted for when performing the surgical closure so as not to apply too much pressure to the wound edges.

Prolene (polypropylene) is a monofilament suture of more recent development. It has extremely low tissue reactivity which is better than nylon. It has slightly more memory than monofilament nylon. However, it has the greatest tensile strength and the best resistance to infection, as shown in several studies. It is manufactured in a clear and a blue suture, this making it easily visible. The knot security with both prolene and the other monofilament sutures can be improved by adding an extra surgical throw at the time of wound closure.

Ethibond (braided, coated polyester) is the newest and most costly suture for skin surgery. It possesses a low tissue reactivity, passes through tissue easily, and has less memory than monofilament sutures so that its workability and know security increases. As with monofilament sutures, there is a low incidence of infection. Therefore, other than its cost, this is an excellent suture for the dermatologic surgeon.

\section{Surgical Needles}

Modern cutaneous surgery does not warrant the use of needles which require threading, produce large holes, and require the passage of a double layer of suture through the wound. Modern suture material is swedged onto the needle to provide for a smaller diameter needle as well as one diameter of suture having to be pulled through tissue.

Various types of tips on needles are available. These are illustrated in Figure 1. In almost all cases of cutaneous surgery today, the reverse cutting needle is preferred. The needle tip has a cutting edge on the outside of the curve. It actually makes a small cut in the tissue to allow smooth passage of needle and suture. Since the cut is made in the direction opposite to the wound edge, the suture does not tear through tissue as sometimes happens with conventional cutting needles with the cutting surface on the inside of the curve. Precision point needles (Fig. 1) offer the highest degree of honing of the steel and points which are reported to maintain sharpness for a longer period of time.

Needle nomenclature has evolved in a haphazard way without long-term planning. The surgeon must learn through experience which needles he prefers and the terminology of the manufacturer of the needle. In our experience, the needles manufactured by Ethicon Corporation have superior sharpness and are particularly able to maintain their sharpness. We, therefore, are familiar with their nomenclature. With the Ethicon nomenclature, the two series of needles used are F series and the P series. The FS code denotes "for skin," the PS needle (-) "plastic skin," and the P needle (-) "plastic." The FS and PS needles are 3/16 of a circle. The former is the least expensive needle and is used for most noncosmetic surgery. The PS needles are made of better steel and honed to a higher degree. The P-series needles are a semicircle, small that the PS series, and represent the least tissue drag. The P-series needles, because of their increased honing and better quality of steel, are used for most cosmetic work. However, the P and PS series cost approximately three times as much as the FS series. Therefore, the economics of the choice of suture and needle becomes important.

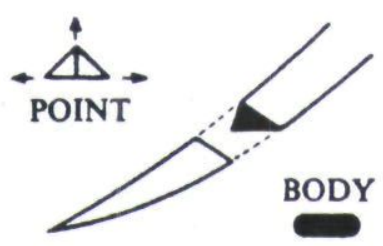

CONVENTIONAL CUTTING

Two opposing cutting edges, with a third on inside curve. Change in cross-section from a triangular cutting tip to a flattened body.

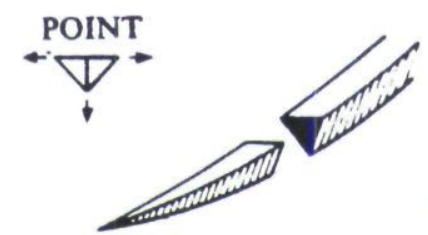

REVERSE CUTTING

Cutting edge on outer curve.

For tough, difficultto-penetrate tissues.

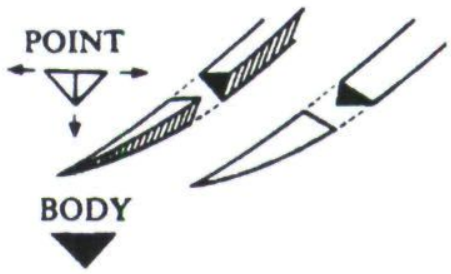

PRECISION POINT

For delicate plastic or cosmetic surgery. Cutting tip, honed 24 extra times.

FIG. 1. Various types of tips on needles used for sutures. 


\section{Wound Management ${ }^{12,13}$}

A basic consideration of the biology of wound healing is important in suture selection. In essence, wound healing can be delineated into three phases: (1) the lag phase from day 0 to day 5 ; (2) the phase of fibroplasia from day 5 to day 14 ; and (3) the phase of maturation from day 14 until wound healing is complete. During the first phase, the wound depends upon sutures to maintain closure and strength. The phase of fibroplasia is a time when a rapid gain in wound strength takes place; however, skin approximation is equally important. Skin approximation usually is complete when maturation begins. This is a phase of connective tissue remodeling and molding. It lasts from four to six weeks. Nature then remolds the wound for approximately eighteen months. Studies have pinpointed that there is increased collagen production in a wound up to day 42 . There probably is no clear distinction between the phases of fibroplasia and maturation as they represent a continuum. In general, wound strength continues to increase for the ensuing year. Experimental studies of skin wounds have shown that although wounds gain strength at relatively early postwound time, they have not yet regained the strength of normal skin. Therefore, it is obvious that some form of wound support is necessary for a much longer period of time than was originally thought. If collagen production continues into the sixth week, then the use of the newer absorbable sutures which maintain tensile strength for close to that period of time should provide improved results. Wound taping and other measures of aiding wound support can also be beneficial.

The choice of suture in a surgical wound, therefore, depends on several factors. Wound healing, as already mentioned, plays an important role. The cleanness (lack of infection) plays a role, as monofilament sutures are better tolerated in a contaminated wound than braided sutures. The anatomic location of the wound is also important. For most dermatologic surgery on areas not around orifices and eyelids, monofilament sutures are perfectly acceptable. However, their memory and rigidity can be a nuisance around eyelids and orifices and areas where pressure is placed on the ends of the suture. Suture size also is an important variable. The size listings on suture range from 0 through $8 / 0$ in sutures used by the dermatologic surgeon. The $8 / 0$ suture is the finest. One should also note that size is dependent on a specific diameter to produce a sufficient tensile strength. Therefore, the diameters of suture for the same size suture will vary. For example, a 5/0 silk suture will be greater in diameter than a $5 / 0$ prolene suture. In general the smaller sutures $(5 / 0,6 / 0)$ are used for cosmetic areas and on the face where the tissue is under the least amount of tension. the $3 / 0$ and $4 / 0$ sutures are used on the torso of the body where the wound is under greater stress. The very strong sutures are used in special circumstances, such as the approximation of galea in scalp reduction. The especially fine sutures $(7 / 0,8 / 0)$ are used for cosmetic procedures performed around eyelids such as blepharoplasties and skin grafts.

Suture removal is another important consideration. Generally, the least amount of time the suture is left in place, the better the cosmetic result. This is also related to the tightness of the suture. Wound edges should be lightly kissed in an everted manner for best tissue approximation. Tying the suture too tight can inhibit this process. Face sutures usually are removed in three to five days. On the neck and other areas under more tension, the sutures are removed five to seven days. On the torso, specifically the back and areas affected by a great deal of motion, the suture is often left in place for two weeks or more. The type of wound closure can also affect the time of suture removal. For example, a wound closed with a running subcuticular stitch can have that stitch left in place for a much longer period of time than a wound closed with interrupted and/or mattress stitches.

We favor the use of monofilament sutures for wound approximation in most instances. This is because of their high tensile strength, low tissue reactivity, and lesser incidence of infection.

\section{Staples ${ }^{13,14}$}

Staples have been commonly used for years to close skin following thyroid surgery. Hunt, ${ }^{12}$ in his classic book on wound healing, states that staples give one of the best experimental wound closures available. They are finding increasing use in general surgery as a skin closure. Stapling is a fast and effective method to close many skin wounds. Staples can be used to hasten skin grafting and the closure of long linear wounds. There are two commonly used staplers.

The Autosuture ${ }^{\circledR}$ is a nondisposable apparatus which is made in both stainless steel and plastic models. They both are autoclavable. A disposable cartridge is available in two widths of staples which is inserted just prior to use. It has a trigger pull mechanism to insert the staple, and a tip which can be turned 360 degrees to facilitate staple insertion. Disposal staple removers are available for staple extraction.

The Proximate ${ }^{\circledR}$ disposable skin stapler is a new apparatus manufactured by Ethicon. It is made predominantly of plastic and contains either 15 or 35 stainless steel staples which are approximately $5.7 \mathrm{~mm}$ in width. This whole unit is disposable. It can be used easily for linear incisions, but because the head does not rotate, it can be difficult to use in hard-to-reach locations. 


\section{Summary}

The development of modern suture material offers the dermatologic surgeon an important choice in would closure. This review, although prejudiced in some instances by the author's experience, likes, and dislikes, is an attempt to clarify the difference between various absorbable and nonabsorbable sutures. Properties of suture material are discussed in detail. Guidelines for choice in regard to wound healing, location of incision, and surgical needles are offered. Lastly, the use of staples in wound closure is mentioned.

\section{References}

1. Van Winkle W, Hastings JC: Considerations in the choice of suture material for various tissues. Surg Gynecol Obstet 135:113, 1972

2. Van Winkle W, Salthouse TN: Biological response to sutures and principles of suture selection, scientific exhibit; American College Surgeons, 1975 Clinical Congress, S.F.
3. Aston SJ: The choice of suture material for skin closure. J Dermatol Surg Oncol 2:57, 1976

4. Herrmann JB: Changes in tensile strength and knot security of surgical sutures in vivo. Arch Surg 106:707, 1973

5. Postlethwait RW, Willigan DA, Ulin AW: Human reaction to sutures. Ann Surg 181:144, 1975

6. Howes EC: Strength studies of polyglycolic acid versus catgut sutures of the same size. Surg Obstet Gynecol 137:18, 1973

7. Horton CE, Adamson JE, Mladick RA, et al: Vicryl synthetic absorbable sutures. Am Surg 40:729, 1974

8. Craig PH, Williams JA, Davis KW, et al: A biologic comparison of polyglactin 910 and polyglycolic acid synthetic abosrbable sutures. Surg Gynecol Obstet 141:1, 1975

9. Katz AR, Turner JR: Evaluation of tensile strength and absorption properties of polyglycolic acid sutures, Surg Gynecol Obstet 701,1970

10. Herrmann JB, Kelley RJ, Higgins GA: Polyglycolic acid sutures. Arch Surg 100:486, 1970

11. Postlethwait RW: Long term comparative study of nonabsorbable sutures. Ann Surg 171:892, 1970

12. Hunt TK, Dunphy JE: Fundamentals of Wound Management, New York Appleton-Century-Crofts, 1979

13. Sato RM, Tebbetts JB, Saarex AJ, et al: Staples: Their use in achieving biological coverage of burn patients. Burns 6:482, 1980

14. Swanson NA, Tromovitch TA, Stegman SJ, et al: The split thickness skin graft, 1980. J Dermatol Surg Oncol 6:524, 1980

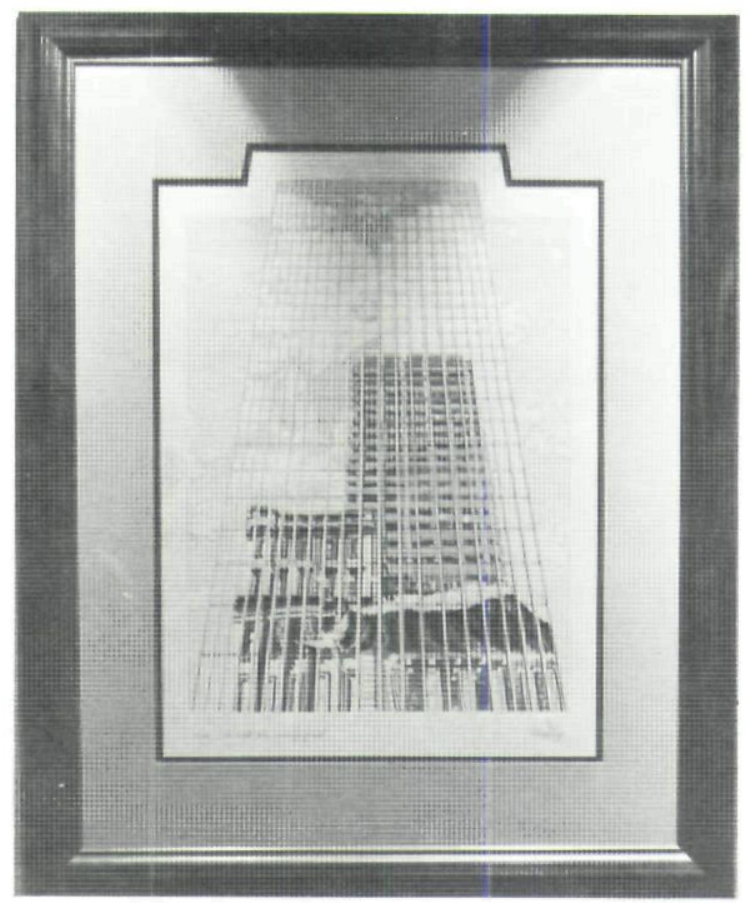

"Alex ... Through the Looking Glass," Mrs. Norman Goldstein. From the 1981 art exhibit of the American Academy of Dermatology, San Francisco, CA. Photograph courtesy of Hoechst-Roussel Pharmaceuticals, Inc. 
This document is a scanned copy of a printed document. No warranty is given about the accuracy of the copy. Users should refer to the original published version of the material. 